\title{
Évaluation de la phase pilote de l'utilisation des tests de diagnostic rapide du paludisme au niveau communautaire, au Burkina Faso
}

\begin{tabular}{|c|c|}
\hline \multicolumn{2}{|c|}{$\begin{array}{l}\text { Authors: } \\
\text { Rachidatou Compaoré }{ }^{1} \\
\text { Adja M. Ouédraogo } \\
\text { Halima Tougri }^{1} \\
\text { Ousmane Badolo } \\
\text { Séni Kouanda }\end{array}$} \\
\hline $\begin{array}{l}\text { Affiliations: } \\
{ }^{1} \text { Biomédical et } \\
\text { Publique, Insti } \\
\text { Recherche en } \\
\text { Santé (IRSS), } \\
\text { Burkina Faso }\end{array}$ & $\begin{array}{l}\text { Santé } \\
\text { tut de } \\
\text { Sciences de la } \\
\text { uagadougou, }\end{array}$ \\
\hline $\begin{array}{l}\text { 2Jhpiego, Oua } \\
\text { Burkina Faso }\end{array}$ & dougou, \\
\hline $\begin{array}{l}{ }^{3} \text { Department } \\
\text { Epidemiology, } \\
\text { Africain de Sa } \\
\text { Burkina Faso }\end{array}$ & $\begin{array}{l}\text { If } \\
\text { Institut } \\
\text { té Publique, }\end{array}$ \\
\hline $\begin{array}{l}\text { Research Proj } \\
\text { Project Numb }\end{array}$ & $\begin{array}{l}\text { ect Registration: } \\
\text { er: } 077\end{array}$ \\
\hline $\begin{array}{l}\text { Correspondin } \\
\text { Rachidatou Cc } \\
\text { rachidoc7@ya }\end{array}$ & $\begin{array}{l}\text { m author: } \\
\text { mpaoré, } \\
\text { hoo.fr }\end{array}$ \\
\hline $\begin{array}{l}\text { Dates: } \\
\text { Received: } 14 \text { S } \\
\text { Accepted: } 27 \\
\text { Published: } 20\end{array}$ & $\begin{array}{l}\text { ept. } 2019 \\
\text { une } 2020 \\
\text { Oct. } 2020\end{array}$ \\
\hline $\begin{array}{l}\text { How to cite th } \\
\text { Compaoré, R., } \\
\text { A.M., Tougri, } \\
\text { Kouanda, S., } 2 \\
\text { 'Évaluation de } \\
\text { de l'utilisation } \\
\text { diagnostic rap } \\
\text { paludisme au } \\
\text { communautai } \\
\text { Faso', African } \\
\text { Journal } 8(1) \text {, a } \\
\text { doi.org/10.41 }\end{array}$ & $\begin{array}{l}\text { is article: } \\
\text { Ouédraogo, } \\
\text { H., Badolo, O. \& } \\
\text { 020, } \\
\text { la phase pilote } \\
\text { des tests de } \\
\text { ide du } \\
\text { niveau } \\
\text { e, au Burkina } \\
\text { Evaluation } \\
\text { 437. https:// } \\
\text { 2/aej.v8i1.437 }\end{array}$ \\
\hline $\begin{array}{l}\text { Copyright: } \\
\text { (C) 2020. The A } \\
\text { Licensee: AOS } \\
\text { work is license } \\
\text { the Creative C } \\
\text { Attribution Lic }\end{array}$ & $\begin{array}{l}\text { uthors. } \\
\text { S. This } \\
\text { d under } \\
\text { ommons } \\
\text { ense. }\end{array}$ \\
\hline Read online: & \\
\hline 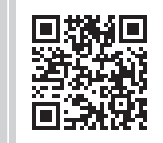 & $\begin{array}{l}\text { Scan this QR } \\
\text { code with your } \\
\text { smart phone or } \\
\text { mobile device } \\
\text { to read online. }\end{array}$ \\
\hline
\end{tabular}

Evaluation of the Pilot Phase of the Use of Rapid Malaria Diagnostic Tests at the Community Level in Burkina Faso:

Introduction: According to the World Health Organization (WHO) guidelines, treatment for malaria should be carried out after diagnostic confirmation. At the community level, diagnostic confirmation by rapid diagnostic tests (RDTs) and treatment of malaria cases should be ensured by Community-Based Health Workers (ASBCs). This formative evaluation thus aimed to measure the level and quality of implementation of the pilot phase of the use of RDTs for malaria case confirmation at the community level in Burkina Faso from 2013 to 2015.

Materials and methods: A multiple case study with multiple levels of analysis was conducted using a concurrent mixed approach in three health districts (Saponé, Nouna and Kaya). The quantitative approach consisted of administering a questionnaire to different groups of stakeholders (ASBCs, beneficiaries and health workers). The qualitative approach combined document review, individual interviews and focus groups with various key informants. The analysis of quantitative data was carried out using Stata version 15 and Excel 2007 software. A thematic content analysis was carried out using Nvivo 10.0 software for the qualitative data. A triangulation of the different quantitative and qualitative data was performed to deepen the analysis and validate some of our results.

Results: The pilot phase of the use of RDTs for malaria at the community level was an intervention that was well accepted by the populations. The different components and activities of the intervention have been implemented. All ASBCs were trained in the use of RDTs. The integration of the supervision of ASBCs into the overall supervision of community activities was a positive aspect of the intervention. However, there were some difficulties in the implementation of each component of the intervention: not all the health and social promotion centres (CSPSs) in the three districts implemented this strategy to the same degree of adherence. In fact, difficulties were noted during the evaluation, such as the lack of RDTs and the existence of outdated stocks at the ASBCs, as well as the lack of security equipment. In addition, the monitoring of the intervention was limited by the poor quality of reporting of the activities carried out, due to the low level of education of most of the ASBCs and the weakness of supervision.

Conclusion: The evaluation of the implementation of case confirmation by community-based RDTs in the health districts of Kaya, Nouna, and Saponé, shows that not all components were implemented with a high degree of adherence to the initial plan. The occurrence of a series of moderating factors that were not adequately supervised could partly explain this performance.

Keywords: Evaluation; Implementation; Malaria; Rapid diagnostic test; RDTs; Community; Burkina Faso.

\section{Introduction}

Malgré les progrès réalisés en matière de prévention, de diagnostic et de prise en charge, l'Organisation Mondiale de la Santé (OMS) estime que 3.2 milliards de personnes environ - soit près de la moitié de la population mondiale - restent exposées au risque de contracter le paludisme. Ainsi, en 2017 dans le monde, on dénombrait 219 millions de cas de morbidité et 438000 de mortalité dus au paludisme (World Health Organization 2018). L'Afrique subsaharienne est, de loin, le continent le plus touché avec $92 \%$ des cas de paludisme et $93 \%$ des décès dus à cette maladie (World Health Organization 2018). 
Au Burkina Faso, selon les statistiques de 2014 du Ministère de la Santé, 8278408 cas ont été notifiés par les formations sanitaires, dont 463774 cas de paludisme grave et 5632 décès. Par rapport aux autres maladies, ces cas représentent $58.4 \%$ des motifs de consultation dans les formations sanitaires de base, $24.8 \%$ des motifs d'hospitalisation et $26.2 \%$ des décès (Ministère de la Santé 2015).

Face à cette situation, le Burkina Faso a souscrit à diverses initiatives de lutte contre le paludisme: l'initiative " Faire reculer le paludisme », la Déclaration d'Abuja et les Objectifs du Millénaire pour le développement (OMD) à travers sa cible $\mathrm{N}^{\circ} 8$, qui visait à maitriser, avant 2015 , le paludisme et d'autres maladies. Dans ce but, diverses stratégies de lutte contre le paludisme, telles que la chimioprévention du paludisme saisonnier, le traitement préventif intermittent chez les femmes enceintes, la pulvérisation intradomiciliaire, les moustiquaires imprégnées d'insecticide à longue durée d'action (MILDA), et la prise en charge du paludisme à domicile (PECADO), ont ainsi été mises en place. La prise en charge précoce et correcte de tout cas confirmé de paludisme dans toutes les formations sanitaires et au niveau communautaire constitue l'une des principales priorités du plan stratégique du Programme National de Lutte contre le Paludisme (PNLP) en cours (Burkina Faso 2015).

En effet, les dernières recommandations de l'OMS préconisent la confirmation parasitologique chez tout patient se présentant avec des signes cliniques compatibles avec le paludisme, à tous les niveaux du système de santé, avant l'administration de tout traitement antipaludique (World Health Organization, Global Malaria Programme 2011).

Cette confirmation parasitologique, qui devrait permettre d'améliorer la qualité des soins, est rendue possible grâce à la disponibilité des tests de diagnostic rapide (TDR) qui, avec des sensibilités de détection du paludisme comparables à la microscopie de routine (Bell 2006; Harvey et al. 2008; Moody 2002; Murray et al. 2003), pourraient améliorer le diagnostic, notamment chez les enfants fébriles, dans les zones touchées par le paludisme (Young 2003). L'utilisation d'outils de diagnostic du paludisme peut potentiellement reduire le gaspillage et le cout pour l'achat des medicaments antipaludéens (Hume et al. 2008), retarder éventuellement le développement de la résistance des parasites aux médicaments et enfin, améliorer le traitement d'autres causes de la fièvre telles que la pneumonie (Msellem et al. 2009; Perkins \& Bell 2008; World Health Organization 2005). Des études menées en Tanzanie (Mubi et al. 2011) et en Zambie (Yeboah-Antwi et al. 2010) ont ainsi montré une diminution de la prescription de combinaisons thérapeutiques à base d'artémisinine (CTA) suite à l'utilisation des TDRS par les agents de santé.

Afin de respecter les recommandations de l'OMS, le PNLP au Burkina Faso a mis en place une intervention sur l'utilisation des TDR par les agents communautaires de santé au niveau de trois districts sanitaires.
Desétudes ont montré queles agents de santé communautaires peuvent, avec une bonne formation, confirmer les cas de paludisme avec précision et en toute sécurité. Il a également été rapporté que cette stratégie est en général bien acceptée par les populations et par les agents de santé dans les formations sanitaires (Mukanga et al. 2010; Mukanga et al. 2012; Zongo, Farquet \& Ridde 2016). Cependant, même si l'importance d'une telle évaluation a déjà été soulignée, il y a peu de données sur la mise en œuvre de cette intervention au niveau communautaire, par des agents de santé à base communautaire (ASBC) (Beaudry \& Gauthier 1992; Champagne, Contandriopoulos \& Denis 1990; Patton 1960, 1997; Rossi \& Freeman 1993).

La plupart des études et évaluations sur la mise en œuvre des TDR en milieu communautaire se sont concentrées sur la faisabilité, l'efficacité et l'acceptation de la stratégie de l'intervention (Msellem et al. 2009; Mubi et al. 2011; YeboahAntwi et al. 2010). Cependant, l'analyse de la fidélité de mise en œuvre aussi est importante, car elle permet de garantir que les résultats sont produits par l'intervention conduite selon les modalités opérationnelles prévues lors de la conception de l'intervention (Elliott \& Mihalic 2004; Perez et al. 2009; Tiono et al. 2008). Toutefois, les évaluations ont souvent négligé ce domaine, ce qui n'a donc pas permis d'apprécier l'écart entre l'efficacité attendue d'un programme reposant sur l'adoption de TDR et son efficacité dans des conditions réelles (Elliott \& Mihalic 2004). Par ailleurs, l'évaluation de la fidélité de mise en œuvre permet également, non seulement d'expliquer le succès ou l'échec des programmes mis en œuvre, mais aussi de générer des idées ayant comme objectif l'amélioration de la mise en œuvre de l'intervention (Perez et al. 2009). Ce dernier point est important, surtout dans cadre d'interventions pilotes qui doivent être mises à l'échelle nationale.

Au Burkina Faso, des lacunes ont déjà été constatées dans la mise en œuvre de certaines interventions du Programme National de Lutte contre le Paludisme (PNLP) (De Allegri et al. 2013; Tiono et al. 2008), compte tenu du contexte de mise en œuvre et de la fragilité du système de santé. Donc, afin de s'assurer de l'effectivité de la mise en œuvre de la phase pilote des TDR au niveau communautaire et de son bon fonctionnement dans tous les districts, le PNLP, en collaboration avec les partenaires techniques et financiers, a conduit deux missions d'évaluation de la phase pilote des TDR communautaires, dans tous les trois districts pilotes, Kaya, Saponé et Nouna. Chacun de ces sites a la particularité d'abriter un site de surveillance démographique et de santé. Cette évaluation se justifie également du fait du caractère pilote de l'intervention, en vue d'un passage réussi à l'échelle nationale.

Certaines études se sont déjà intéressées à l'analyse de la fidélité de mise en œuvre des interventions communautaires contre le paludisme au Burkina Faso, comme la distribution de moustiquaires imprégnées d'insecticides à longue durée d'action (MILDA) (Ridde et al. 2013) ou la chimioprévention du paludisme saisonnier (CPS) (Compaoré et al. 2017), mais 
elles restent rares. Ces évaluations se sont confrontées à de nombreux problèmes et défis tels que le chevauchement des actions, surtout en présence d'autres projets/programmes simultanément mis en œuvre, l'interférence de facteurs contextuels lors de la mise en œuvre et le grand nombre d'adaptations retrouvées dans la mise en œuvre. En outre, la plupart de ces études ont utilisé des approches purement qualitatives pour évaluer le degré de fidélité de mise en œuvre des programmes et elles se sont concentrées sur un ou deux sites de mises en œuvre de l'intervention désignée, ce qui rend souvent difficile la validité externe des conclusions correspondantes.

L'objectif de cette évaluation formative était d'apprécier la fidélité de la mise en œuvre de l'intervention au niveau de ces trois districts sanitaires pilotes, deux ans après le début de la mise en œuvre, afin de contribuer à améliorer de façon continue l'implantation de l'intervention, lors du passage à l'échelle nationale.

L'évaluation avait pour objectif de mesurer spécifiquement l'adhésion des principales composantes de l'intervention au plan initial de mise en œuvre, selon trois dimensions de la fidélité à savoir le contenu, la couverture et la temporalité, en conformité avec le cadre conceptuel de mesure de la fidélité proposé par Hasson (Hasson, Blomberg \& Dunér 2012), adapté de celui de Caroll et al. (2007). Afin d'intégrer toute la complexité de l'intervention dans cette évaluation, les auteurs de cet article, qui ont fait partie de l'équipe d'évaluation, ont adopté une approche mixte à la fois quantitative et qualitative, afin de pouvoir conduire une analyse approfondie prenant en compte toutes les caractéristiques de l'intervention, et de façon comparable dans les trois sites.

\section{Méthodologie \\ Cadre de l'évaluation}

Le Burkina Faso présente un système sanitaire de type pyramidal à trois niveaux. Le district sanitaire (DS) constitue la porte d'entrée du système, chaque DS étant dirigé par une équipe cadre de district (ECD) et relevant d'une direction régionale de la santé (DRS). Le système sanitaire comporte plusieurs centres de santé et de promotion sociale (CSPS) qui sont dirigés par des infirmiers chefs de poste (ICP).

Cette évaluation a été conduite dans trois districts sanitaires au Burkina Faso: (1) le DS Kaya dans la région du CentreNord avec une superficie de $5584 \mathrm{~km}^{2}$ et une population estimée à 564867 habitants en 2014; (2) le DS de Nouna dans la région du Boucle du Mouhoun, couvrant une superficie de $7424 \mathrm{~km}^{2}$, et comptant 358695 habitants; et (3) le DS de Saponé dans la région du Centre-Sud avec une superficie de 1700 km² $^{2}$ et 106502 habitants, en 2016 (Ministère de la Santé 2015).

Ces districts ont été choisis au regard de leur situation géographique (chaque district est situé à proximité d'un site de surveillance épidémiologique et démographique) et au regard de leur expérience en matière de prise en charge à domicile (PECADO) du paludisme.

La phase pilote de l'intervention s'est déroulée de mars 2013 à août 2015.

\section{Description de la stratégie d'intervention}

L'objectif visé par le PNLP à travers cette intervention était de confirmer et de traiter correctement, avec des antipaludiques, tous les cas suspects de paludisme simple au niveau communautaire, par les ASBC.

Les principales composantes de cette intervention étaient les suivantes:

- La communication en faveur de l'utilisation des TDR à travers:

- Un plaidoyer à l'endroit des autorités, des leaders et des Partenaires Techniques et Financiers (PTF)

- Une mobilisation sociale

- Une Communication pour le Changement de Comportement (CCC)

- L'approvisionnement et la gestion des intrants: TDR, CTA et kits d'administration

- Le renforcement des compétences des responsables de formations sanitaires, des agents de santé et des ASBC

- La confirmation des cas suspects avec les TDR

- Le suivi/évaluation de l'utilisation des TDR en milieu communautaire.

\section{Type d'évaluation}

L'évaluation s'est fondée sur des études de cas multiples, à plusieurs niveaux d'analyse. Chaque cas était représenté par un district sanitaire et les niveaux d'analyse étaient constitués par les dimensions de la fidélité ainsi que des composantes de l'intervention. Une enquête transversale a été conduite suivant une approche mixte quantitative et qualitative concomitante, dans les districts sanitaires de Kaya, Saponé et Nouna.

\section{Typologies de parties prenantes incluses dans l'échantillon de l'évaluation}

L'évaluation a concerné les acteurs de mise en œuvre de l'intervention et la population bénéficiaire des trois DS.

Pour l'enquête quantitative, des responsables au niveau des directions régionales de la santé (DRS) dont relèvent les DS, ainsi que des ECD et CSPS, et des ASBC ont été sélectionnés de manière raisonnée en fonction de l'information souhaitée. La répartition des enquêtés est décrite dans le Tableau 1 présenté dans la section sur les constats. Pour la population bénéficiaire, une stratégie d'échantillonnage aléatoire simple a été adoptée en utilisant la base de données des districts sanitaires pour sélectionner le nombre de participants nécessaire pour l'étude quantitative. La taille de l'échantillon a été estimée à l'aide de la formule pour une proportion

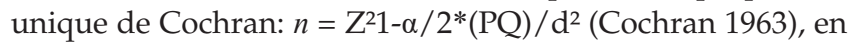


TABLEAU 1: Échantillon de l'évaluation.

\begin{tabular}{|c|c|c|c|c|c|c|}
\hline \multirow[t]{2}{*}{ Type d'enquête } & \multirow[t]{2}{*}{ Méthode de collecte } & \multirow[t]{2}{*}{ Cibles } & \multicolumn{3}{|c|}{ Effectifs } & \multirow[t]{2}{*}{ Tota } \\
\hline & & & $\begin{array}{c}\text { DS/Kay } \\
\text { A }\end{array}$ & $\begin{array}{c}\text { DS/Noun } \\
a\end{array}$ & $\begin{array}{c}\text { DS/Sapon } \\
\text { É }\end{array}$ & \\
\hline \multirow[t]{5}{*}{ Enquête quantitative } & Enquête & ECD & 1 & 1 & 1 & 3 \\
\hline & & DRS & 1 & 1 & 1 & 3 \\
\hline & & Infirmiers chefs de poste(ICP)/responsable des stocks & 6 & 5 & 5 & 16 \\
\hline & & Bénéficiaires & 152 & 164 & 171 & 487 \\
\hline & & Agents de santé communautaires & 12 & 10 & 10 & 32 \\
\hline \multirow[t]{6}{*}{ Enquête qualitative } & Entretien individuel & Membre de l'ECD & 1 & 1 & 1 & 3 \\
\hline & Entretien individuel & ICP & 6 & 5 & 5 & 16 \\
\hline & Entretien individuel & Directeur régional de santé & 1 & 1 & 1 & 3 \\
\hline & Focus group & Bénéficiaires (Femmes) & 1 & 1 & 1 & 3 \\
\hline & Focus group & Bénéficiaires (Hommes) & 1 & 1 & 1 & 3 \\
\hline & Focus group & Leaders communautaires & 1 & 1 & 1 & 3 \\
\hline
\end{tabular}

$\overline{E C D}$, équipe-cadre de district; $\mathrm{DRS}$, direction régionale de la santé.

considérant une erreur d'échantillonnage de 5\%, un niveau de confiance de $95 \%$, un recours aux services des ASBC de $50 \%$. Un taux de non-réponse de $10 \%$ a été autorisé.

Pour l'enquête qualitative, des informateurs clés ont été sélectionnés de manière raisonnée parmi les acteurs de mise en œuvre et les bénéficiaires de l'intervention. Plusieurs focus groups ont été également réalisés auprès de trois groupes constitués chacun de femmes, d'hommes et de leaders communautaires dans chaque district. L'échantillon de l'enquête qualitative est décrit dans le Tableau 1, présenté dans la section sur les constats.

\section{Description de l'échantillon}

L'enquête a concerné 3 membres des DRS, dont relève chaque DS de l'évaluation, 3 membres des ECD de chaque DS, 161 infirmiers chefs de poste ICP, 37 ASBC et 487 membres de la population cible bénéficiaire de l'intervention de l'aire sanitaire de 31 CSPS. Parmi les 487 bénéficiaires enquêtés dans les ménages, nous retrouvons 152 personnes provenaient du DS de Kaya, 164 du DS de Nouna.

Plus de détails sur l'échantillon de l'évaluation sont fournis dans le tableau ci-dessous.

\section{Collecte et traitement des données}

La collecte a duré 11 jours dans les DS sanitaires et 3 semaines au niveau central; elle a été effectuée par des agents de collecte préalablement formés.

Plusieurs techniques et outils de collecte ont été utilisés auprès des différents groupes de répondants:

- En ce qui concernel'enquête quantitative, un questionnaire a été utilisé et administré dans les ménages.

- Pour l'enquête qualitative, une revue des documents stratégiques et de gestion de l'intervention a tout d'abord été conduite. Des entretiens individuels ont été ensuite effectués auprès des informateurs clés impliqués dans la mise en œuvre à différents niveaux. Dans chaque district, 3 focus groups de 8 à 10 personnes ont été réalisés auprès des hommes et des femmes ayant bénéficié des services des ASBC, ainsi que de leaders communautaires.

- Les entretiens individuels et les focus groups ont été menés en français ou en langue locale.

- Des guides d'entretiens semi-structurés spécifiques à chaque catégorie de répondant et des supports de focus group ont servi pour les entretiens qualitatifs; pour les entretiens qualitatifs. Les donnees ont ete enregistrees à l'aide d'un dictaphone après le consentement des participants, et complétées par une prise de notes.

- Pour l'enquête qualitative, les entretiens ont porté sur le rôle des agents de santé dans les DS, les ECD et les responsables des directions régionales de la santé (DRS) dans la mise en œuvre de la stratégie et leurs perceptions sur la mise en œuvre proprement dite (processus de mise en œuvre de la stratégie, facteurs modérateurs et contextuels). Le questionnaire administré à la population cible de l'intervention a concerné leurs connaissances sur les TDR et leur accès à la confirmation par les TDR au niveau communautaire.

\section{Analyse des données}

L'analyse des données quantitatives a été réalisée à l'aide des logiciels Stata version 15 et Excel 2007. Les variables qualitatives ont été exprimées en nombre et en pourcentage, et les variables quantitatives en moyenne et écart-type.

Pour les données qualitatives, une analyse de contenu thématique a été effectuée avec l'appui du logiciel Nvivo 10.0. Tous les entretiens qualitatifs ont été transcrits, traduits en français et saisis sous MS Word. Pour assurer la qualité des transcriptions et éviter toute déformation des propos, les enregistrements ont été confiés à des professionnels ayant une maîtrise parfaite de la langue locale ainsi que du français. Les données ont ensuite été dépouillées suivant un codebook décrivant les thématiques d'intérêts de l'étude.

Dans la phase pré-évaluative, une liste exhaustive des activités planifiées a été établie (annexe 1), lors de la reconstitution de la théorie de changement du projet au cours des discussions autour de l'évaluabilité de l'intervention. 
Les activités retenues ont ensuite été classées en fonction des cinq composantes principales de l'intervention. Cela a été possible grâce à une revue approfondie de documents officiels et administratifs ainsi qu'à de nombreux entretiens avec des acteurs clés sélectionnés au sein d'un échantillon raisonné des personnes ressources.

Chaque enquêté(e) a été invité(e) à réagir sur le degré de mise en œuvre, non seulement des activités planifiées mais aussi de celles modifiées ou ajoutées.

Une triangulation des résultats des données quantitatives et qualitatives a été réalisée pour approfondir l'analyse et valider certains de nos résultats.

Les données recueillies ont été étudiées sur la base d'un cadre d'analyse adapté concernant la fidelité de mise en oeuvre, qui a été intialement proposé par Carroll et al., modifié par Hasson (Carroll et al. 2007; Hasson, Blomberg \& Dunér 2012).

Les différents niveaux d'analyse ont concerné les trois dimensions de l'évaluation de la fidélité de mise en œuvre (contenu, couverture et calendrier) ainsi que toutes les composantes de la stratégie (communication pour un changement de comportement, approvisionnement et gestion des intrants, renforcement des compétences des agents de santé et des ASBC, confirmation des cas avec les TDR par les ASBC et le suivi-évaluation).

Les données de fidélité d'implantation du contenu et de calendrier des activités recueillies qualitativement lors des entretiens individuels ont été traduites en données quantitatives en considérant la proportion d'activités classées par les informateurs clés selon quatre modalités:

- l'activité était implantée comme planifiée (I)

- l'activité était implantée comme planifiée ou modifiée (I/M)

- l'activité était adaptée et/ou modifiée (M)

- l'activité était «non implantée » $(\mathrm{N})$.

Ces données ont été ensuite présentées sous forme de graphiques. Ces modèles de quantification et de représentation graphiques ont été adoptés de la classification de Ridde et al., adaptée de Perez (Perez et al. 2009; Ridde et al. 2013). Quant aux données concernant l'adhésion du contenu à la fidélité de mise en œuvre, elles concernaient les pourcentages de la population cible atteinte par les activités de formation, de sensibilisation et de confirmation des cas de paludisme par les ASBC.

La Figure 1 illustre le degré de fidelité de la mise en en oeuvre de différentes composantes de l'intervention dans les trois districts sanitaires (I= Implanté comme prévu; I/M= Implanté mais avec des modifications: $\mathrm{M}=$ Modifié; $\mathrm{N}=$ Modifié avec l'ajout de nouvelles activités).

\section{Considérations éthiques}

Le protocole a été soumis à l'examen du Comité d'Éthique pour la Recherche en Santé du Burkina Faso (CERS) et l'approbation du CERS a été obtenue (N²016-6-077) avant la conduite de cette étude. Afin d'assurer le respect des

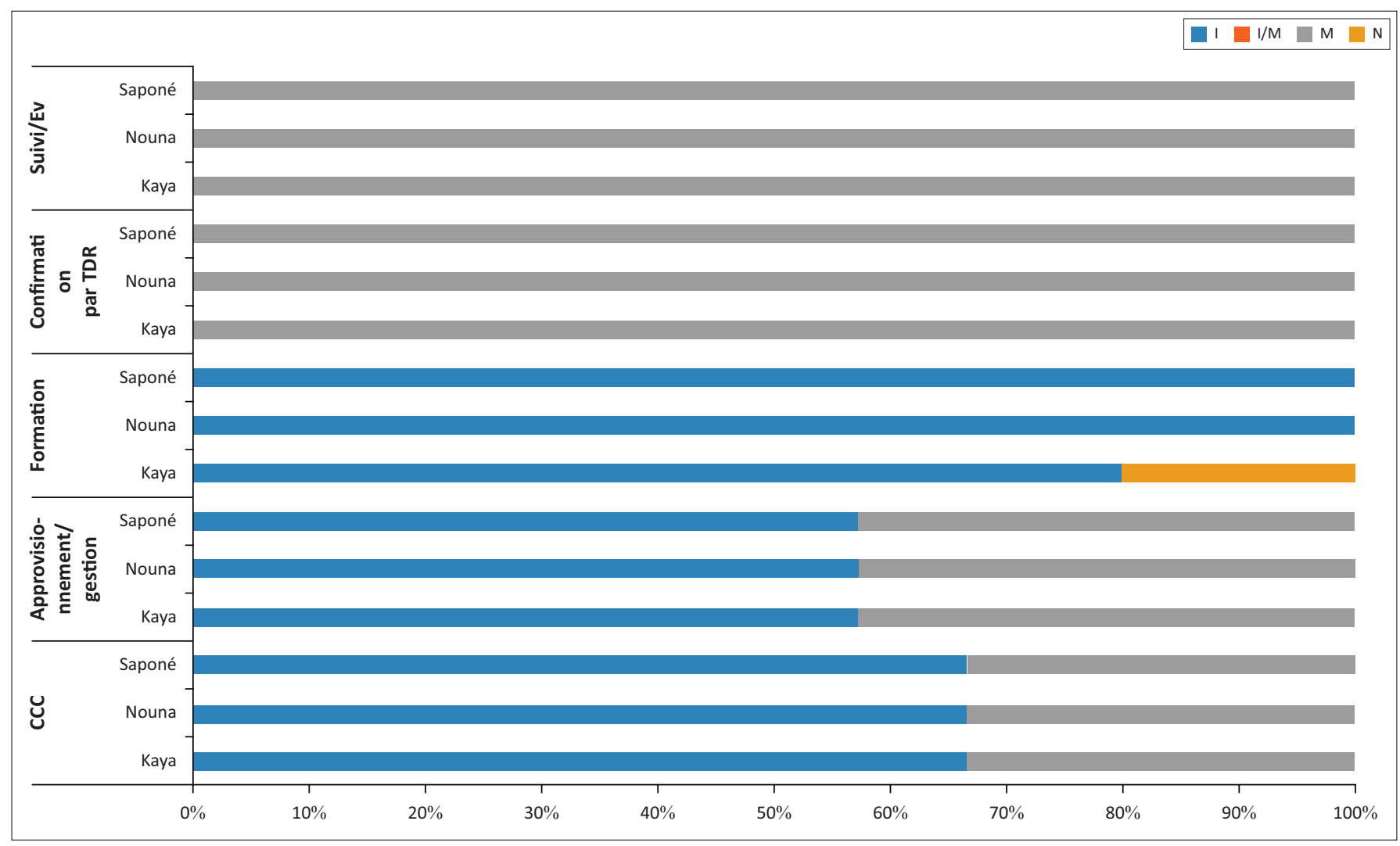

FIGURE 1: Adhésion du contenu à la fidélité de mise en œuvre. 
principes éthiques, l'équipe de recherche a été particulièrement attentive au respect $\mathrm{du}$ consentement éclairé à l'inclusion dans l'étude, à l'anonymat et au respect de la confidentialité.

\section{Conclusions}

\section{Adhésion du contenu à la fidélité de mise en ouvre}

D'une manière générale, la mise en œuvre semble homogène dans les trois districts pour les différentes composantes de l'intervention.

\section{Communication pour un changement de comportement}

La composante sensibilisation a adhéré dans son contenu au même degré de fidélité dans les trois DS. Les activités mises en œuvre ont respecté le plan initial à plus de 6\%. La population était en général informée de la stratégie.

\section{Formation}

La composante "formation » est celle qui a le mieux adhéré à la mise en œuvre avec 100\% d'adhésion à Saponé et Nouna et $80 \%$ à Kaya. Les propos recueillis confirment que les ICP et les ASBC ont bénéficié d'un renforcement de compétence en cascade, tel que prévu dans le plan initial:

"... il faut reconnaitre que nous, on a été formés au district sanitaire de Saponé sur la prise en charge du paludisme au niveau communautaire; en retour nous avons rencontré les $A S B C$ des différents villages là pour les former. » Agent de santé, DS Saponé.

Les ASBC confirment également avoir reçu une formation sur la réalisation des TDR. C'est ce qu'a confié cet ASBC de Nouna:

\begin{abstract}
«Nous avons été formés avant de commencer. Au cours de la formation, nous avons appris comment échanger avec la personne avant de faire le prélèvement, comment amener la personne à accepter, et comment repérer des signes lorsqu'un enfant a la fièvre. C'est ce que nous avons appris.» ASBC, DS Nouna.
\end{abstract}

\section{Approvisionnement/gestion des stocks}

La composante " approvisionnement/gestion des stocks ", avec un niveau d'exécution à $57 \%$, a connu le plus faible degré d'adhésion à la fidélité.

Les DS et les ASBC ont été approvisionnés en TDR et en outils de gestion/suivi (cahier de consultation et de gestion des médicaments) au début de l'intervention, par le niveau central. Cependant, Nous avons noté une insuffisance dans la gestion de ces stocks du point de vue de la conservation et de la gestion. Au début de la phase pilote, il n'y a pas eu de dotation en consommables (gants, boîtes de sécurité), comme le souligne cet ASBC:

«...Tout le matériel n'est pas au complet. Oui, parce que c'est nous qui nous nous débrouillons pour avoir les gants. Il faut tout faire pour que le matériel soit disponible, puisque pendant la formation on nous a dit que le prélèvement de sang peut être source de contamination par certaines maladies, mais c'est nous-mêmes qui cherchons les gants » ASBC, DS Saponé.

La totalité des DS (100.0\%) et la quasi-totalité (94.7\%) des CSPS disposaient d'outils de gestion de stocks tandis que $56.8 \%$ des ASBC n'en possédaient pas. La majorité des stocks disponibles chez les ASBC lors du passage des enquêteurs étaient mal conservés dans des cartons, entreposés à même le sol.

Quant aux ruptures de stock, il faut noter qu'au niveau central, il n'y a pas eu de rupture de stock durant la phase pilote. Cependant, près de $67 \%$ des ASBC avaient connu une rupture au cours de cette période. Lors du passage des enquêteurs, il est ressorti que plus du quart des ASBC (48.0\%), 83.3\% des CSPS et 33.3\% des DS étaient en rupture de stock. L'analyse des rapports a montré que certains ASBC n'ont été dotés qu'une seule fois en TDR, tandis que d'autres n'ont jamais reçu de dotation dans un des CSPS pilotes.

Paradoxalement, il a été retrouvé sur le terrain des ASBC qui avaient des stocks de TDR périmés. Les procédures de gestion des TDR périmés mis en place n'étaient pas non plus respectées: les produits périmés étaient déposés à même le sol; le retrait de stocks de TDR périmés un mois avant la date de péremption et sa destruction sur place n'étaient pas respectés.

Les principales causes de péremption rapportées étaient surtout les erreurs dans les prévisions, mais aussi un faible recours aux ASBC, dans certains villages.

\section{Confirmation des cas par les tests de diagnostic rapide}

En ce qui concerne la composante « confirmation des cas par les TDR » par les ASBC, aucune activité à ce niveau n'a été mise en œuvre telle qu'elle était initialement prévue. Pour la réalisation des TDR, les ASBC ont été confrontés à une difficulté liée au séchage du diluant des TDR, en raison des conditions climatiques et de stockage des intrants. Plus du tiers des TDR ont été réalisés au CSPS (31\% à Kaya, $42 \%$ à Nouna et $44 \%$ à Saponé); du fait de l'instauration de la politique de gratuité des soins pour les enfants de moins de cinq ans, la population préférait se rendre directement au CSPS, d'une part parce que certains ASBC faisaient payer les gants à 75 FCFA par TDR, d'autre part parce que, du fait de la rupture en CTA, ils auraient à réaliser le TDR deux fois de suite, une fois par l'ASBC, et une autre fois par l'agent de santé au CSPS. Cependant, en raison d'une rupture de stocks de TDR dans les CSPS, certains patients qui s'étaient rendus directement dans le centre de santé, se voyaient renvoyer chez les ASBC pour la confirmation par TDR.

\section{Suivi-évaluation}

Pour la composante « suivi-évaluation » également, les activités ont été pour la plupart modifiées. Du fait de contraintes financières, la supervision a été intégrée dans la routine des CSPS. Cependant, les agents de santé ne 
disposaient pas de canevas pour la supervision des ASBC. Environ 31.4\% des ASBC n'avaient jamais administré de TDR à un patient en présence d'un superviseur. Près de $89 \%$ des ASBC soumettaient un rapport mensuel d'activités aux ICP. Cependant, ces rapports étaient peu informatifs dans leur contenu en raison de la faible qualité du rapportage: les outils de suivi étaient mal tenus et très peu mis à jour chez plus du tiers des ASBC (135.5\%) et dans la majorité des CSPS (52.6\%) et des DS $(66.7 \%)$ :

"...le circuit de transmission n'était pas tout à fait clair dans les esprits...souvent il faut que l'ICP même se déplace pour aller chercher $l^{\prime} A S B C$, ils vont s'assoir d'abord dans sa case (de l'ASBC), pour faire son rapport (rapport de l'ASBC), avant que l'ICP ne rentre avec l'information. Au lieu que le rapport ne monte de l'ASBC à l'ICP, c'est l'ICP qui part chercher le rapport; et quand tu as beaucoup de villages, il faut comprendre que c'est quasi impossible... " Agent de santé, Kaya.

\section{Adhésion de la couverture des activités à la fidélité de mise en œuvre}

L'évaluation de l'adhésion de la couverture des activités à la fidélité de mise en œuvre a concerné le degré d'atteinte des agents de santé ciblés par la formation et le degré d'atteinte des bénéficiaires par l'intervention. En ce qui concerne la formation, les taux de couverture chez les ICP étaient élevés à Kaya et à Nouna, respectivement à $135 \%$ et $91 \%$. Les données n'ont pas été retrouvées pour le DS de Nouna. La totalité des ASBC avait reçu une formation sur l'utilisation des TDR et la prise en charge du paludisme simple dans les trois DS. La couverture des agents déjà formés et qui devaient être recyclés était un peu plus faible, surtout à Nouna (66.7\%). Les enquêtés ont déclaré avoir bénéficié, eux-mêmes ou un membre de leur famille, de la confirmation par un TDR d'un cas suspect de paludisme, réalisée par les ASBC; ainsi, les cas suspects de paludisme ont été confirmés respectivement à $90 \%$ à Kaya et $69 \%$ à Saponé, contre seulement 55\% dans le DS de Nouna. Cela s'explique par le fait que le DS de Nouna couvre une superficie plus grande avec des zones difficilement accessibles géographiquement.

\section{Adhésion du calendrier à la fidélité de mise en œuvre}

L'évaluation de l'adhésion du calendrier à la fidélité de mise en œuvre a concerné essentiellement les composantes suivantes: «formation », " approvisionnement et gestion des stocks » et le "suivi évaluation » des activités.

Le calendrier des activités planifiées ont été jugées soit implantées ou modifiées (I/M), soit modifiées (M) dans l'ensemble.

\section{Formation}

Selon les agents de santé, le renforcement des compétences des ASBC avait duré trois jours:

"... Ils ont été formés durant les trois jours avec des exercices pratiques ». Agent de santé, DS Kaya.
Cependant, la durée moyenne de la formation des ASBC était de 5 jours avec des extrêmes de 1 à 7 jours, selon les rapports d'activités.

Pour les ASBC ayant reçu un suivi post-formation, la durée moyenne du suivi était de 2 jours avec des extrêmes de 1 à 7 jours.

\section{Approvisionnement}

Les délais de livraison n'ont pas été respectés tels que cela avait été prévu depuis l'acquisition des intrants jusqu'à la dotation des ASBC.

Le dispositif de ravitaillement des ASBC en TDR a connu des débuts difficiles, notamment dans le district de Kaya:

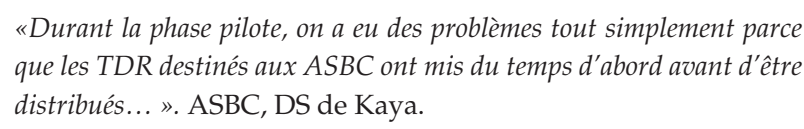
que les TDR destinés aux ASBC ont mis du temps d'abord avant d'être distribués... ». ASBC, DS de Kaya.

$\mathrm{Au}$ niveau central, la CAMEG n'a pas pu respecter le délai d'acquisition des TDR. Ce qui a eu une répercussion sur le reste des activités liées à l'approvisionnement à tous les niveaux (DRS,DS,CSPSetcommunauté).La fréquencederenouvellement des stocks n'a pas non plus été respectée. Les principales causes de péremption des TDR étaient essentiellement les erreurs de prévision et les délais de péremption courts, avec des stocks qui étaient abondants. La livraison avec une date de péremption proche a été retrouvée au niveau des DS, des CSPS et chez les ASBC à respectivement $33.3 \%, 6.3 \%$ et $20.3 \%$ :

" ...la date de péremption déjà posait problème... " Agent de Santé, Kaya.

\section{Suivi évaluation}

La fréquence des supervisions n'a pas été respectée à tous les niveaux.

De façon générale, les fréquences de supervision étaient peu nombreuses et très irrégulières dans tous les trois districts. Les raisons invoquées du non-respect du calendrier prévu étaient les mêmes dans les trois DS: l'insuffisance du personnel de santé, la grande superficie de l'aire sanitaire des CSPS, le manque de moyen de transport ou l'insuffisance de ressources financières pour une supervision rapprochée. Ainsi, selon un agent de santé du DS de Nouna:

\footnotetext{
«... les activités programmées c'était la supervision, mais en fin 2015 on n'arrivait pas à faire toutes les supervisions parce qu'on avait un problème de la logistique roulante; aussi quand il commence à pleuvoir il y a les contraintes naturelles. Par exemple il y a un village à 2 kilomètres quand il commence à pleuvoir, pour y accéder il faut nager. Donc il y a les contraintes naturelles qui sont là. C'est pour ça que certaines supervisions n'ont pas été faites».
}

\section{Conclusions préliminaires}

L'intérêt d'une évaluation de la mise en œuvre d'une intervention, qui permettrait d'expliquer le succès ou l'échec de programmes implantés dans leur contexte réel, n'est plus 
à démontrer (Perez et al. 2009; Ridde et al. 2013). Cela est d'autant plus nécessaire dans le cas du PNLP, au vu des nombreux challenges auxquels le programme a dû faire face dans les expériences de mise en œuvre et de passage à l'échelle de d'intervention antérieures visant à lutter contre le paludisme (De Allegri et al. 2012, 2013; Ridde et al. 2013; Tiono et al. 2008).

Les résultats de cette évaluation ont montré que la mise en œuvre de la confirmation par les TDR de tout cas suspect de paludisme dans les trois districts sanitaires a été effective. Presque toutes les activités qui avaient été planifiées initialement ont été réalisées. Cependant, de nombreuses modifications ont été notées chez quasiment toutes les composantes de l'intervention et dans les trois districts sanitaires, à des degrés assez semblables. Cela pourrait être dû aux mêmes difficultés contextuelles rencontrées dans les interventions précédentes, comme les faiblesses du système de santé en termes de manque de ressources humaines, matérielles et financières, et les défis liés au recours aux agents de santé à base communautaire. En effet, le faible niveau d'instruction des ASBC et la nature volontaire de leur implication dans les stratégies de lutte contre le paludisme constituent souvent des entraves dans la mise en œuvre des interventions de santé en Afrique.

\section{Adhésion du contenu à la fidélité de mise en œuvre}

Les composantes qui ont subi le plus de modifications dans leur contenu ont été surtout la réalisation des TDR par les ASBC devant tout cas suspect de paludisme, le suivi/ évaluation des activités en milieu communautaire et la gestion de la chaîne d'approvisionnement des intrants de l'intervention. La plupart de ces changements ont été observés dans la pluralité des sites où l'évaluation s'est déroulée. Ces modifications peuvent être liées à la complexité de l'intervention elle-même, mais semblent avoir été plus liées au contexte de mise en œuvre de l'intervention. En effet, du point de vue technique, les ASBC ne semblaient pas éprouver de difficultés particulières à la réalisation des TDR dans leur communauté. Cela a sûrement été facilité d'une part par la formation dont ils ont bénéficié avant le début de l'intervention, mais également à leur expérience dans la prise en charge du paludisme à travers la PECADO. Ces facteurs semblent donc avoir agi plus comme des facteurs facilitant la mise en œuvre de l'intervention. Par contre, d'autres facteurs liés au contexte ont rendu difficile cette mise en œuvre. Certaines de ces difficultés, telles que le climat très chaud et sec des zones d'implantation de l'intervention et la faiblesse du système de santé en ressources nécessaires à la conduite d'activités comme le suivi, auraient pu être anticipées et corrigées dès la phase de planification. D'autres par contre, comme l'adoption assez rapide et au plan national en 2015 de la politique de gratuité des soins pour les enfants de moins de cinq ans, qui a modifié les comportements de recours aux soins de la population cible de l'intervention, semblent avoir été assez inattendues. Cependant, l'effet de la gratuité des soins sur le recours aux soins de la population avait déjà été noté dans certaines zones comme Kaya, avec l'expérience de l'ONG "Save the children ». Cette constatation confirme ce qui avait déjà été rapporté dans la littérature sur le fait qu'une intervention de santé publique n'évolue pas en vase clos, mais interagit avec son contexte de mise en œuvre, dont il faudrait tenir compte, si l'on veut atteindre un degré élevé de fidélité à la mise en œuvre (Dusenbury et al. 2003; Pérez et al. 2016; Von Thiele Schwarz, Hasson \& Lindfors 2015). Cela s'est également ressenti dans le degré de couverture de la cible par les activités.

\section{Adhésion de la couverture à la fidélité de mise en œuvre}

Les taux de couverture des agents par la formation est appréciable dans les trois districts; cependant, le taux de confirmation par TDR de tous les cas suspects de paludisme en milieu communautaire est loin d'atteindre les 100\% souhaités, surtout dans les districts sanitaires de Saponé et Nouna. Cela est sans doute une conséquence des difficultés déjà rapportées, dues aux facteurs contextuels. Dans le cas du district sanitaire de Nouna, ces difficultés ont été plus accentuées du fait que l'aire du district sanitaire est plus vaste et certaines zones plus difficiles d'accès. Cela a compliqué encore plus l'approvisionnement en intrants des différents acteurs et la supervision de leurs activités par les ICP, ce qui aurait pu permettre de corriger certains facteurs susceptibles d'avoir influencé négativement la mise en œuvre de l'intervention.

Toutefois, au vu des nombreuses limitations notées dans la gestion des stocks de TDR, il conviendrait de renforcer la formation sur ce point dans tous les districts sanitaires, pour une meilleure rationalisation des ressources et une plus grande efficacité de l'intervention.

Les problèmes rencontrés dans le respect des délais ont sans doute contribué à la faible couverture de la confirmation des cas en milieu communautaire.

\section{Adhésion du calendrier à la fidélité de mise en œuvre}

Les résultats de l'évaluation montrent que la plupart des activités, dont la fréquence et la durée ont été évaluées, ont subi des modifications. Si certaines études ont montré qu'une certaine modification était nécessaire pour une adaptation de l'intervention à un environnement contraignant, un certain niveau d'adhésion à la fidélité de mise en œuvre demeure cependant nécessaire et cette adaptation devrait toutefois respecter la théorie du changement pour l'atteinte des objectifs visés (Pérez et al. 2016; Von Thiele Schwarz, Hasson \& Lindfors 2015). Dans notre évaluation, la plupart des modifications observées dans le respect du calendrier ont été surtout dues à la faiblesse des ressources et à la gestion de l'intervention entre les différents acteurs (partenaires financiers, centrale d'achat et de gestion des médicaments génériques (CAMEG), PNLP, DS, CSPS et ASBC) pour la gestion des intrants. En effet, si dans les plans initiaux, la supervision de la mise en œuvre de la confirmation en milieu 
communautaire par les TDR devait être intégrée à la supervision périodique des districts sanitaires, dans la réalité, les activités des ASBC étaient peu, ou rarement supervisées par les agents de santé, dû au manque de ressources pour ces activités. Cela a joué un rôle important dans la qualité de la mise en œuvre, qui est un autre facteur de modération dans la fidélité de mise en œuvre. Ce défaut d'intégration a ainsi créé une situation complexe et floue, ayant conduit au gaspillage des ressources. Cette supervision était en effet bien nécessaire car, malgré leur expérience, les ASBC ont été confrontés à certaines difficultés comme le stockage correct et la gestion des intrants. Même si il a été rapporté dans la littérature que les ASBC ayant reçu une formation sont en mesure d'effectuer et d'interpréter correctement les TDR, même après un long temps, il a été aussi attesté que la faiblesse de la supervision ne permet pas de juger de la qualité de la mise en œuvre, ni de la compliance aux recommandations de traitements (Dusenbury et al. 2003; Mukanga et al. 2012; Von Thiele Schwarz, Hasson \& Lindfors 2015). Singlovic et al avaient rapporté qu'une bonne formation et une supervision régulière étaient associées à un faible taux de non compliance aux résultats des TDR (Singlovic et al. 2016). Le manque de données dans cette évaluation ne nous permet pas de conclure sur une éventuelle amélioration de la qualité des soins grâce à l'utilisation des TDR en milieu communautaire. Des inquiétudes avaient déjà été soulevées par certains auteurs quant à l'efficacité de l'intervention à améliorer la qualité des soins dans les systèmes de santé africains actuels (English et al. 2009).

Cette situation a soulevé de nombreux débats dans la littérature en santé mondiale. Des études ont suggéré que certains programmes ciblés de lutte contre certaines maladies pourraient même affaiblir les systèmes de soins de santé dans les pays à faible revenu en créant des systèmes de prestation parallèles superflus et en perturbant les activités quotidiennes du système de santé (Atun et al. 2010; Belaid et al. 2017; Marchal et al. 2011; Travis et al. 2004). Si certains auteurs affirment qu'une grande partie des avantages des interventions sanitaires verticales sont perturbés par le bouleversement des services de santé généraux et par les inefficacités causées par le manque d'intégration entre les différents services de santé, il est cependant reconnu que le débat sur l'efficacité relative des programmes verticaux et horizontaux se déroule à un degré de généralité si élevé qu'il est difficile d'en tirer des conclusions solides; par ailleurs, de nombreux systèmes de santé existants tendent à associer les programmes horizontaux et verticaux, ce qui rend difficile l'évaluation de leur efficacité relative (Atun et al. 2010; Marchal et al. 2011; Travis et al. 2004). Ce manque d'intégration réelle, en tant que facteur clé, pose par ailleurs la question de la pérennisation de certains programmes de santé.

\section{Apport de l'évaluation de la fidélité de mise en œuvre}

$\mathrm{Au}$ regard de tous ces constats, la présente évaluation souligne encore une fois la nécessité de la prise en compte du contexte, lors de la mise en œuvre des interventions et du renforcement des services de santé pour un suivi effectif et efficace, afin de corriger les éventuels écarts à la fidélité pouvant entraver le succès de la présente intervention.

Le cadre conceptuel utilisé pour l'analyse a aussi permis de pouvoir mesurer l'adhésion à la fidélité des activités de l'intervention par rapport à son plan initial, tout en soulignant l'importance des facteurs contextuels dans la mise en œuvre d'interventions complexes (Hasson, Blomberg \& Dunér 2012).

\section{Apport de l'utilisation des méthodes mixtes dans l'analyse de fidélité de mise en œuvre}

Les études de cas réalisées avec les méthodes mixtes dans le cadre de cette évaluation se sont avérées particulièrement bien adaptées au but de cette évaluation car la complexité de l'intervention exigeait une combinaison de méthodes afin de pouvoir répondre à chacune des questions posées par l'évaluation. Ainsi, l'enquête quantitative nous a permis essentiellement de pouvoir estimer la dimension " couverture » de la fidélité. Quant aux données qualitatives, elles ont permis non seulement de renseigner les niveaux de mise en œuvre des activités, mais aussi à travers leur quantification, de pouvoir comparer les degrés de fidélité entre les trois districts sanitaires. Les données qualitatives ont en outre permis une triangulation efficace par rapport aux inférences se basant sur les données quantitatives et, pour ce faire, en passant par la valorisation des perceptions des différents acteurs de mise en œuvre, elles ont fait ressortir les facteurs influençant la fidélité de mise en œuvre (Belaid et al. 2017). Cette approche a donc permis une complémentarité des données, grâce à l'augmentation de l'exhaustivité des constats de l'évaluation à travers l'utilisation de différentes méthodes. Cela a enfin entrainé une meilleure compréhension de l'intervention et une analyse plus approfondie. Tout cela a accru la validité et la crédibilité des conclusions de l'évaluation.

Cette évaluation démontre donc non seulement l'importance d'apprécier la fidélité de mise en œuvre, mais aussi la pertinence d'explorer les innovations méthodologiques dans le domaine de l'évaluation des interventions de santé publique. En effet, elle permet d'accroître le nombre des évaluations fondée sur une approche de méthodes mixtes dans le domaine de la santé, et dans le même temps, de supporter l'usage du qualitatif au-delà du rapportage des perceptions des informateurs-clefs (Belaid et al. 2017).

\section{Limites de l'évaluation}

L'existence des sites de surveillance a été d'une grande aide dans cette évaluation pour apprécier l'atteinte de la population cible par les différentes activités de l'intervention. Cependant, des biais de mémoire ont sûrement pu entacher la qualité de cette évaluation, notamment en ce qui concerne les activités dont les aspects temporels ont fait l'objet des entretiens avec les parties prenantes. Toutefois, la triangulation des sources des données a été poursuivie afin 
de minimiser ces biais. Par ailleurs, par manque de ressources, il n'a pas été possible, dans cette évaluation, d'apprécier la performance des ASBC dans la réalisation des TDR.

\section{Conclusions finales}

L'évaluation de la mise en œuvre de la confirmation des cas par les TDR en milieu communautaire dans les districts sanitaires de Kaya, Nouna et Saponé, montre des niveaux différents de degré d'adhésion à la fidélité en fonction des dimensions de la fidélité et selon les composantes de l'intervention. Les difficultés observées sur le terrain se sont révélées assez semblables dans les trois districts et, comme attesté par les évaluations antérieures sur les interventions mises en œuvre par le PNLP, elles soulignent la faiblesse du système de santé. Il est, par conséquent, nécessaire d'y répondre de façon adéquate lors du passage à l'échelle nationale. Cette évaluation enfin démontre l'importance de l'évaluation de la fidélité de mise en œuvre, tout en apportant sa contribution aux approches méthodologiques mixtes dans le domaine de l'évaluation des interventions complexes dans le domaine de la santé publique.

\section{Remerciements Intérêts concurrents}

L'auteur déclare ne pas avoir de relations financières ou personnelles susceptibles de l'avoir influencé de manière inappropriée dans la rédaction de cet article.

\section{Contributions d'auteurs}

A.M.O. et S.K. ont participé à la conception et à la mise en œuvre de l'évaluation de l'intervention. Le premier projet a été rédigé par R.C. et A.M.O. Tous les auteurs ont révisé et approuvé la version finale du manuscrit.

\section{Le financement}

Cette recherche est issue des données d'une évaluation financée par JhPIEGO pour le compte du Programme National de Lutte contre le Paludisme/Ministère de la Santé du Burkina Faso. Le bailleur financier n'a joué aucun rôle dans la conception de l'étude, la collecte et l'analyse des données, la décision de publier ou la préparation du manuscrit.

\section{Déclaration de disponibilité des données}

Le partage de données n'est pas applicable à cet article car aucune nouvelle donnée n'a été créée ou analysée dans cette étude.

\section{Avertissement}

Les points de vue et opinions exprimés dans cet article n'engagent queleurs auteurs et ne reflètent pas nécessairement la politique ou la position officielles des agences affiliées à leurs auteurs.

\section{Références}

Atun, R., De Jongh, T., Secci, F., Ohiri, K. \& Adeyi, O., 2010, 'Integration of targeted health interventions into health systems: A conceptual framework for analysis', Health Policy Plan 25(2),104-111. https://doi.org/10.1093/heapol/czp055

Beaudry, J. \& Gauthier, B., 1992, 'L'évaluation de programme', Rech Soc Problématique À Collecte Données 425-452.

Belaid, L., Bodson, O., Oumar, S., Valéry, R. \& Anne-Marie, T.-T., 2017, 'Nouvelles contributions de l'approche qualitative dans l'évaluation des interventions en santé mondiale', Cah Réalis 12.

Bell, D., Wongsrichanalai, C. \& Barnwell, J.W., 2006, 'Ensuring quality and access for malaria diagnosis: How can it be achieved?', Nature Reviews Microbiology 4(9), 682-695. https://doi.org/10.1038/nrmicro1474

Burkina Faso, Ministère de la santé, Programme Nationale de Lutte Contre le Paludisme (PNLP), 2015, Plan Stratégique National 2014-2017, Ouagadougou.

Carroll, C., Patterson, M., Wood, S., Booth, A., Rick, J. \& Balain, S., 2007, 'A conceptual framework for implementation fidelity', Implementation Science 2(1), 40. https:// doi.org/10.1186/1748-5908-2-40

Champagne, F., Contandriopoulos, A.-P. \& Denis, J.-L., 1990, La recherche évaluative en santé, Groupe de recherche interdisciplinaire sur la santé, Université de Montréal.

Cochran, W.G., 1963, Sampling technique, 2nd edn., John Wiley and Sons Inc., New York, NY.

Compaoré, R., Yameogo, M.W.E., Millogo, T., Tougri, H. \& Kouanda, S., 2017, 'Evaluation of the implementation fidelity of the seasonal malaria chemoprevention intervention in Kaya health district, Burkina Faso', PLoS One 12(11).

De Allegri, M., Louis, V.R., Tiendrébeogo, J., Souares, A., Yé, M., Tozan, Y. et al., 2013 'Moving towards universal coverage with malaria control interventions: Achievements and challenges in rural Burkina Faso', International Journal of Health Planning and Management 28(1), 102-121. https://doi.org/10.1002/ hpm. 2116

De Allegri, M., Ridde, V., Louis, V.R., Sarker, M., Tiendrebéogo, J., Yé, M. et al., 2012, 'The impact of targeted subsidies for facility-based delivery on access to care and equity - Evidence from a population-based study in rural Burkina Faso', Journal of Public Health Policy 33(4), 439-453. https://doi.org/10.1057/jphp.2012.27

Dusenbury, L., Brannigan, R., Falco, M. \& Hansen, W.B., 2003, 'A review of research on fidelity of implementation: Implications for drug abuse prevention in school settings', Health Education Research 18(2), 237-256. https://doi.org/10.1093/ her/18.2.237

Elliott, D.S. \& Mihalic, S., 2004, 'Issues in disseminating and replicating effective prevention programs', Prevention Science 5(1), 47-53. https://doi.org/10.1023/ Brevention programs, Preventior

English, M., Reyburn, H., Goodman, C. \& Snow, R.W., 2009, 'Abandoning presumptive antimalarial treatment for febrile children aged less than five years - A case of running before we can walk?', PLoS Medicine 6(1), e1000015. https://doi. org/10.1371/journal.pmed.1000015

Harvey, S.A., Jennings, L., Chinyama, M., Masaninga, F., Mulholland, K. \& Bell, D.R. 2008, 'Improving community health worker use of malaria rapid diagnostic tests in Zambia: Package instructions, job aid and job aid-plus-training', Malaria Journal 7, 160. https://doi.org/10.1186/1475-2875-7-160

Hasson, H., Blomberg, S. \& Dunér, A., 2012, 'Fidelity and moderating factors in complex interventions: A case study of a continuum of care program for frail elderly people in health and social care', Implementation Science 7(23), 1-11. https://doi.org/10.1186/1748-5908-7-23

Hume, J.C.C., Barnish, G., Mangal, T., Armázio, L., Streat, E. \& Bates, I., 2008 'Household cost of malaria overdiagnosis in rural Mozambique', Malaria Journal 7, 33. https://doi.org/10.1186/1475-2875-7-33

Marchal, B., Van Dormael, M., Pirard, M., Cavalli, A., Kegels, G. \& Polman, K., 2011, 'Neglected tropical disease (NTD) control in health systems: The interface between programmes and general health services', Acta Tropica 120, S177-S185. between programmes and general health services',
https://doi.org/10.1016/j.actatropica.2011.02.017

Ministère de la Santé, 2015, Annuaire statistique santé 2014, Ouagadougou, Burkina Faso, p. 330, viewed 13 January 2016, from http://www.cns.bf/IMG/pdf/ annuaire_2014_du_ms.pdf.

Moody, A., 2002, 'Rapid diagnostic tests for malaria parasites', Clinical Microbiology Reviews 15(1), 66-78. https://doi.org/10.1128/CMR.15.1.66-78.2002

Msellem, M.I., Mårtensson, A., Rotllant, G., Bhattarai, A., Strömberg, J., Kahigwa, E., et al., 2009, 'Influence of rapid malaria diagnostic tests on treatment and health outcome in fever patients, Zanzibar: A crossover validation study', PLoS Medicine 6(4), e1000070. https://doi.org/10.1371/journal.pmed.1000070

Mubi, M., Janson, A., Warsame, M., Mårtensson, A., Källander, K., Petzold, M.G., et al., 2011, 'Malaria rapid testing by community health workers is effective and safe for targeting malaria treatment: Randomised cross-over trial in Tanzania', PLoS One 6(7), e19753. https://doi.org/10.1371/journal.pone.0019753

Mukanga, D., Tibenderana, J.K., Kiguli, J., Pariyo, G.W., Waiswa, P., Bajunirwe, F. et al., 'Community acceptability of use of rapid diagnostic tests for malaria by community health workers in Uganda', Malaria Journal 9(1), 203. https://doi. org/10.1186/1475-2875-9-203

Mukanga, D., Tibenderana, J.K., Peterson, S., Pariyo, G.W., Kiguli, J., Waiswa, P. et al., 2012, 'Access, acceptability and utilization of community health workers using diagnostics for case management of fever in Ugandan children: A cross-sectional study', Malaria Journal 11(1), 121. https://doi.org/10.1186/1475-2875-11-121 
Murray, C.K., Bell, D., Gasser, R.A. \& Wongsrichanalai, C., 2003, 'Rapid diagnostic testing for malaria', Tropical Medicine \& International Health 8(10), 876-883. https://doi.org/10.1046/j.1365-3156.2003.01115.x

Patton, M.Q., 1960, Qualitative evaluation and research methods, Sage, Newbury Park, CA.

Patton, M.Q., 1997, Utilisation-focused evaluation: The new century text, Util Focus Eval News Century Text.

Perez, D., Lefevre, P., Castro, M., Sanchez, L., Toledo, M.E., Baly, A. et al., 2009, 'Fidelity research assists in evaluation, adjustment and scaling-up of community-based interventions in Cuba', in 6th European Congress on Tropical Medicine and International Health. 1st Mediterranean Conference on Migration and Travel Health, pp. 167-167, viewed 13 April 2016, from https://biblio.ugent.be/ publication/935352.

Pérez, D., Van der Stuyft, P., Zabala, M.C., Castro, M. \& Lefèvre, P., 2016, 'A modified theoretical framework to assess implementation fidelity of adaptive public health interventions', Implementation Science 11(1), 91. https://doi.org/10.1186/ s13012-016-0457-8

Perkins, M.D. \& Bell, D.R., 2008, 'Working without a blindfold: The critical role of diagnostics in malaria control', Malaria Journal 7(Suppl 1), S5. https://doi. org/10.1186/1475-2875-7-S1-S5

Ridde, V., Druetz, T., Poppy, S., Kouanda, S. \& Haddad, S., 2013, 'Implementation fidelity of the national malaria control program in Burkina Faso', PLoS One 8(7), e69865. https://doi.org/10.1371/journal.pone.0069865

Rossi, P.H. \& Freeman, H.E., 1993, 'Program monitoring for evaluation and management', Evaluation System Approach 163-213.

Singlovic, J., Ajayi, I.O., Nsungwa-Sabiiti, J., Siribié, M., Sanou, A.K., Jegede, A.S. et al., 2016, 'Compliance with malaria rapid diagnostic testing by Community Health Workers in 3 malaria-endemic countries of sub-Saharan Africa: An observationa study', Clinical Infectious Diseases 63(Suppl 5), S276-S282. https://doi.org/ 10.1093/cid/ciw626
Tiono, A.B., Kaboré, Y., Traoré, A., Convelbo, N., Pagnoni, F. \& Sirima, S.B., 2008, 'Implementation of home based management of malaria in children reduces the work load for peripheral health facilities in a rural district of Burkina Faso', Malaria Journal 7(1), 201. https://doi.org/10.1186/1475-2875-7-201

Travis, P., Bennett, S., Haines, A., Pang, T., Bhutta, Z., Hyder, A.A. et al., 2004, 'Overcoming health-systems constraints to achieve the millennium development goals', The Lancet 364(9437), 900-906. https://doi.org/10.1016/S0140-6736(04)16987-0

Von Thiele Schwarz, U., Hasson, H. \& Lindfors, P., 2015, 'Applying a fidelity framework to understand adaptations in an occupational health intervention', Work (Reading, Mass) 51(2), 195-203. https://doi.org/10.3233/WOR-141840

World Health Organization, 2005, 'Malaria, roll back. Global estrategic plan roll back malaria 2005-2015', in Global estrategic plan roll back malaria 2005-2015, RBM, viewed 08 January 2019, from https://scholar.google.com/scholar lookup?title= Global $\% 20$ Strategic $\% 20$ Plan $\% 3 \mathrm{~A} \% 20$ Roll $\% 20$ Back $\% 20$ Malaria $\% 20$ $2005 \%$ E2\%80\%932015\&publication_year=2005.

World Health Organization, 2018, World malaria report 2018, World Health Organization, Geneva.

World Health Organization, 2011, Universal access to malaria diagnostic testing: An operational manual, World Health Organization, Geneva.

Yeboah-Antwi, K., Pilingana, P., Macleod, W.B., Semrau, K., Siazeele, K., Kalesha, P. et al., 2010, 'Community case management of fever due to malaria and pneumonia in children under five in Zambia: A cluster randomized controlled trial', PLOS Medicine 7(9), e1000340 https://doi.org/10.1371/journal. pmed.1000340

Young, M.W., 2003, 'Effective management of childhood malaria at the community level: Program experience to guide the research agenda', Pap WHOTDR Sci Work Group Malar, Geneva, 24-27 March.

Zongo, S., Farquet, V. \& Ridde, V., 2016, 'A qualitative study of health professionals' uptake and perceptions of malaria rapid diagnostic tests in Burkina Faso', Malaria Journal 15(1), 190. https://doi.org/10.1186/s12936-016-1241-6 